\title{
INFLUÊNCIA DA TEMPERATURA NA BIOLOGIA DE TRIATOMÍNEOS.XX. Triatoma maculata (ERICHSON, 1848) (HEMIPTERA, REDUVIIDAE)
}

\author{
Ionizete Garcia da Silva ', Fernando de Freitas Fernandes", Heloisa Helena Garcia da \\ Silva"
}

\section{RESUMO}

Estudou-se o efeito da temperatura no ciclo evolutivo de Triatoma maculata (Erichson,1848) para estabelecer a sua influência no desenvolvimento do triatomíneo, conhecer alguns aspectos de sua biologia $\mathrm{e}$ dominar técnicas de criação para viabilizar o uso dessa espécie nos testes experimentais em doença de Chagas. O período médio de incubação dos ovos de T.maculata, para machos e fêmeas, foi de 29 e de 16 dias, à temperatura de $25^{\circ}$ e $30^{\circ} \mathrm{C}$, respectivamente. $\grave{A}$ temperatura de $25^{\circ} \mathrm{C}$, a duração média do ciclo evolutivo, para machos e fêmeas, foi, respectivamente, de 223,5 e 222,3 dias e, de 168,7 e 167,2 dias, a $30^{\circ} \mathrm{C}$. Os experimentos foram realizados a $25 \pm 0,5^{\circ} \mathrm{C}$ e $30 \pm 1,0^{\circ} \mathrm{C}$, com umidade de $70 \pm 5 \%$ e fotoperíodo de 12 horas.

UNITERMOS: Triatoma maculata .Biologia. Vetores. Doença de Chagas. Tripanosomíase americana.

\section{INTRODUÇÃO}

Triatoma maculata é a segunda espécie vetora mais importante na Venezuela. Coloniza-se nos domicílios e é comumente encontrada infectada com Trypanosoma cruzi.Ocorre predominantemente no peridomicílio, geralmente associada a ninhos de aves, currais e em pedreiras.No meio silvestre, tem sido encontrado em ninhos de pássaros, principalmente o de Phacellodomus rufifrons inornatus associado a Psammolestes arthuri.

Distribui-se no Brasil (Roraima); Colômbia; Antilhas (Aruba,Bonaire, Curaçao); Guianas; Suriname e Venezuela (LENT \& WYGODZINSKY, 1979).

Com o estudo do desenvolvimento de T.maculata em laboratório, espera-se contribuir para o esclarecimento da sua biologia, nas ações de vigilância entomológica e ainda fornecer subsídios para facilitar a sua criação em laboratório, possibilitando o uso dessa espécie nos testes experimentais em doença de Chagas.

\footnotetext{
Profs. do Depart. ${ }^{\circ}$ de Parasitologia - IPTSP/UFG

"* Farmacêutica do Dept. ${ }^{\circ}$ de Parasitologia - IPTSP/UFG

Apoio financeiró: SENESU, FUNAPE, PRPPG-UFG

Recebido para publicação em 31/05/95
} 


\section{MATERIAL E MÉTODOS}

\section{Origem dos triatomíneos}

A criação de T.maculata iniciou-se a partir de triatomíneos provenientes do Instituto Oswaldo Cruz.

$\mathrm{O}$ desenvolvimento de ovo a adulto realizou-se em frascos de polietileno, cilindricos e transparentes ( $7 \times 3,8 \mathrm{~cm})$, idênticos aos mencionados por SILVA (1985).

Os frascos foram acondicionados em duas câmaras climatizadas, a $25 \pm 0,5^{\circ} \mathrm{C}$ e $30 \pm 1{ }^{\circ} \mathrm{C}$, com umidade de $70 \pm 5 \%$ e fotoperíodo de 12 horas (SILVA \& SILVA, 1988).

\section{Incubação, Ninfas e Adultos}

Os ovos coletados no mesmo dia eram colocados em placas de Petri, forradas com papel filtro, para a incubação. Estas, eram colocadas em caixas de papelão, nas câmaras climatizadas, em ambiente escuro, para evitar a interferência da luz na incubação.

Após a eclosão, as ninfas foram individualizadas em frascos de $3 \times 5 \mathrm{~cm}$, permanecendo nestes, até o $4^{\circ}$ estádio, sendo transferidas para frascos de $3,8 \times 7 \mathrm{~cm}$, onde permaneciam do $5^{\circ}$ estádio até a fase de adulto, quando eram acasalados.

\section{Alimentação}

Os triatomíneos foram alimentados em aves (Gallus gallus domesticus) através da técnica desenvolvida por SILVA (1985). À temperatura de $30^{\circ} \mathrm{C}$, alimentaram-se as ninfas de $1^{\circ}$ estádio, no $8^{\circ}$ dia após a eclosão das mesmas, e, as de $2^{\circ}, 3^{\circ}, 4^{\circ}$ e $5^{\circ}$ estádios, com intervalos de $12,15,20$ e 25 dias, respectivamente, após a ecdise.Os adultos foram alimentados 10 dias após a emergência. A $25^{\circ} \mathrm{C}$, as ninfas de $1^{\circ}$ estádio alimentaram-se no $10^{\circ} \mathrm{dia}$; para os estádios subsequentes e para os adultos, os intervalos foram maiores em 5 dias do que a $30^{\circ} \mathrm{C}$.

\section{Duração dos estádios ninfais e do período ninfal}

Determinou-se a duração dos estádios através das observações das exúvias, que eram recolhidas diariamente.

SILVA, 1.G.; FERNANDES, F.F. SILVA, H.H.G. Influência da temperatura na biologia de triatomíneos.XX Triatoma maculata (Erichson, 1848) (Hemiptera, Reduviidae) Rev. Pat. Trop.24 (1): 49-54, jan/jun. 1995

\section{Análise estatística}

Para as durações do período de incubação dos ovos e dos estádios ninfais, determinaram-se as médias e os respectivos erros-padrão para cada temperatura. A análise da variância foi usada para comparar os dados obtidos nos experimentos realizados a $25^{\circ}$ e a $30^{\circ} \mathrm{C}$.

\section{RESULTADOS}

A duração média do período de incubação dos ovos a $25^{\circ}$ e a $30^{\circ} \mathrm{C}$ foi, respectivamente, de 29 e de 16 dias (Fig. 1), sendo estas médias significativamente diferentes entre si.

Pela análise da Tabela 1, verificam-se diferenças significativas na duração de todos os estádios e do período ninfal, entre $25^{\circ}$ e $30^{\circ} \mathrm{C}$, ao nível de $5 \%$.

TABELA 1. Duração média dos estádios ninfais e do período ninfal, para machos e fêmeas, de Triatoma maculata, às temperaturas de $25^{\circ}$ e de $30^{\circ} \mathrm{C}$ (dias).

\begin{tabular}{lccccc}
\hline \multirow{2}{*}{ Estádio } & \multicolumn{3}{c}{$25^{\circ} \mathrm{C}$} & & \multicolumn{2}{c}{$30^{\circ} \mathrm{C}$} \\
\cline { 2 - 3 } \cline { 5 - 6 } \cline { 5 - 6 } & Macho & Fêmea & & Macho & Fêmea \\
\cline { 2 - 3 } & $25,8 \pm 0,10 \mathrm{a}$ & $25,8 \pm 0,09 \mathrm{a}$ & & $19,9 \pm 0,07 \mathrm{c}$ & $19,8 \pm 0,09 \mathrm{c}$ \\
$1^{\circ}$ & $32,1 \pm 0,28 \mathrm{a}$ & $31,8 \pm 0,28 \mathrm{a}$ & & $22,1 \pm 0,07 \mathrm{c}$ & $22,2 \pm 0,09 \mathrm{c}$ \\
$3^{\circ}$ & $42,7 \pm 0,32 \mathrm{a}$ & $43,3 \pm 0,34 \mathrm{a}$ & & $29,5 \pm 0,11 \mathrm{c}$ & $29,3 \pm 0,10 \mathrm{c}$ \\
$4^{\circ}$ & $40,3 \pm 0,24 \mathrm{a}$ & $40,1 \pm 0,23 \mathrm{~b}$ & & $42,0 \pm 0,15 \mathrm{c}$ & $40,6 \pm 0,14 \mathrm{~d}$ \\
$5^{\circ}$ & $53,8 \pm 0,41 \mathrm{a}$ & $52,3 \pm 0,23 \mathrm{~b}$ & & $42,0 \pm 0,15 \mathrm{c}$ & $40,6 \pm 0,14 \mathrm{~d}$ \\
Período ninfal & $194,6 \pm 0,41 \mathrm{a}$ & $193,3 \pm 0,24 \mathrm{~b}$ & & $152,7 \pm 0,11 \mathrm{c}$ & $151,5 \pm 0,11 \mathrm{~d}$ \\
\hline
\end{tabular}

OBS.: As médias seguidas da mesma letra não apresentam diferenças significativas entre si. 
Verificam-se diferenças significativas na duração do $5^{\circ}$ estádio e do período ninfal a $25^{\circ} \mathrm{C}$, e do $4^{\circ}$ e $5^{\circ}$ estádios e do período ninfal a $30^{\circ} \mathrm{C}$, entre machos e fêmeas, ao nível de $1 \%$. quanto a $30^{\circ} \mathrm{C}$

A Fig. 1 mostra que a duração média aumenta com o estádio, tanto a $25^{\circ}$

O período médio do ciclo evolutivo de Triatoma maculata, para machos e fêmeas, foi, respectivamente, de 223,5 e 222,3 dias, a $25^{\circ} \mathrm{C}$, e de 168,7 e 167,2 dias, a $30^{\circ} \mathrm{C}$, o que corresponde a uma redução no ciclo, pela temperatura, de $24,61 \%$, para machos, e de $24,78 \%$, para fêmeas.

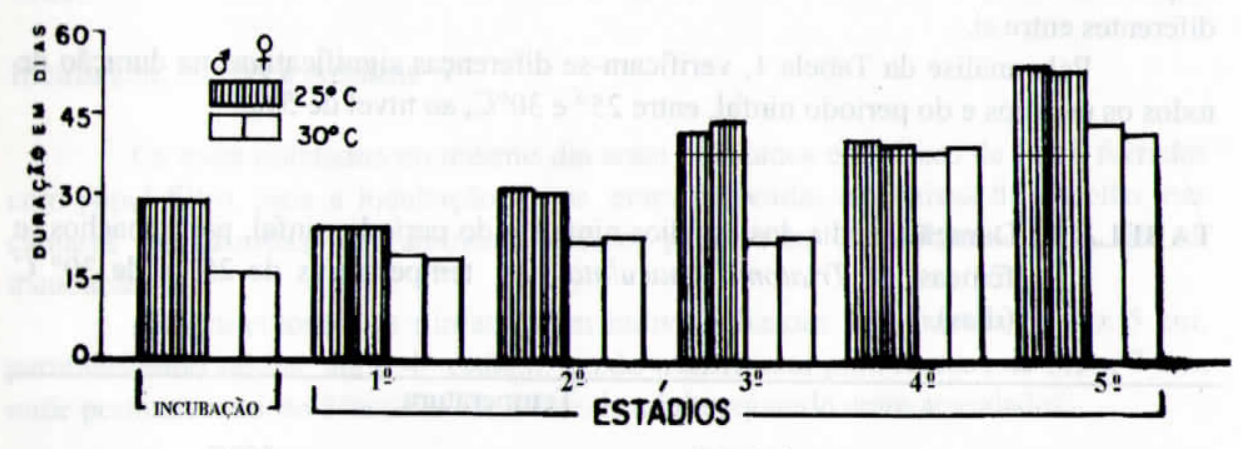

Fig. 1. Duração do período de incubação e dos estádios ninfais de Triatoma maculata, para machos e fêmeas, às temperaturas de $25^{\circ} \mathrm{e}$ de $30^{\circ} \mathrm{C}$.

\section{DISCUSSÃo}

O estudo de mecanismos físicos no desenvolvimento de insetos, como a temperatura, tem mostrado influência básica em seu ciclo de vida (BURSEL, 1964; WIGGLESWORTH, 1972; SILVA, 1985; SILVA \& SILVA, 1988, 1989 e 1993). Neste trabalho, estudou-se dois gradientes de temperaturas e ficou evidente a influência térmica no desenvolvimento de T.maculata, no qual o período de desenvolvimento reduziu cerca de $25 \%$, da temperatura de $25^{\circ} \mathrm{C}$ para $30^{\circ} \mathrm{C}$, passando a ter duas gerações anuais. Estes aspectos, são extremamente positivos na compreensão epidemiológica dessa espécie, para regiões situadas em diferentes faixas isotérmicas. São importantes
SILVA, I.G.; FERNANDES, F.F. SILVA, H.H.G. Influência da temperatura na biologia de triatomineos XX Triatoma maculata (Erichson, 1848) (Hemiptera, Reduviidae) Rev. Pat. Trop.24 (1): 49-54, jan/jun. 1995

também, na produção de triatomíneos em laboratório, pois, além de estimular outras experiências, com novos gradientes de temperatura, sinaliza a possibilidade de produção massal de triatomíneos, cada vez em menores períodos de desenvolvimento e maior economia, colaborando desta forma, na vibilização da pesquisa científica que, neste momento, passa por dificuldades de financiamentos na área universitária, além de servir de treinamento para a iniciação científica.

O desenvolvimento de T.maculata foi idêntico ao de Panstrongylus megistus (SILVA \& SILVA, 1993), nas mesmas condições de temperatura, umidade e fotoperíodo.

\section{SUMMARY}

The influence of temperature on the biology of triatoninae.XX. Triatoma maculata (Erichson, 1848) (Hemiptera, Reduviidae).

The temperature effect was studied at the evolutive cycle of Triatoma maculata to establish your influence at development of the triatomines, to know some aspects of your biology and to dominate creations technics to avaible the use of these species at the experimental tests in Chagas disease. The mean incubation period for males and females, was, 29 and 16 days at $25^{\circ} \mathrm{C}$ and $30^{\circ} \mathrm{C}$, respectively. The mean duration of the developmental cycle for males and females, was, respectively, of 223,5 and 222,3 days at $25^{\circ} \mathrm{C}$, and of 168.7 and 167,2 days at $30^{\circ} \mathrm{C}$. The experiments were performed in two climatized chamber $\left(25^{\circ} \mathrm{C}\right.$ and $\left.30^{\circ} \mathrm{C}\right)$, both with $70 \pm 5 \%$ RU and fotoperiod of 12 hours.

KEYWORDS: Triatoma maculata.Biology. Vectors. Chagas' disease. american tripanosomiasis.

\section{AGRADECIMENTOS}

Ao Dr. José Jurberg, por ter cedido os espécimes de $T$. maculata que deram origem a série em estudo. 
SII.VA, I.G.; FERNANDES, F.F.; SILVA, H.H.G. Influência da temperatura na biologia de triatomíneos.XX. Triatoma maculata (Erichson, 1848) (Hemiptera, Reduviidae) Rev. Pat. Trop.24 ( 1 ): 49 - 54, jan/jun. 1995

\section{REFERÊNCIAS BIBLIOGRÁFICAS}

01.BURSELL, E. Environmental aspects: Temperature, p. 283-321.Indivíduo: M.Rockstein.The Physiology of insecta.V.1., 1964

02.LENT, H. \& Wigodzinsky, P. Revision of the triatominae (Hemiptera, Reduviidae), and theirs significance as vectors of Chagas' disease. Bull.Am.Mus.Nat.Hist.,163: 127-520, 1979

03.SILVA, I.G. da Influência da temperatura na biologia de triatomíneos. I. Triatoma rubrovaria (Blanchhard, 1843) (Hemiptera, Reduviidae). Rev. Goiana Med.,31:1-37, 1985.

04.SILVA, I.G. da \& SILVA, H.H.G. da Influência da temperatura na biologia de triatomíneos. IV. Triatoma infestans (Klug, 1834) (Hemiptera,Reduviidae). An.Soc.Ent.Brasil,17:443-454, 1988.

05.SILVA, I.G. da \& SILVA, H.H.G. da Influência da temperatura na biologia de triatomíneos.IX.Rhodnius nasutus Stal, 1859 (Hemiptera, Reduviidae). Mem.Int.Oswaldo Cruz., 84:377-382, 1989.

06.SILVA, I.G.da \& SILVA, H.H.G. da Influência da temperatura na biologia de triatomíneos. III.Panstrongylus megistus (Burmeister, 1835) (Hemiptera, Reduviidae).Rev.bras.Ent., 37:489-496, 1993.

07.WIGGLESWORTH, V.B. The principles of insect physiology. New York. Halsted. 741 p.1972. 